\title{
How To Find Many Collisions of 3-Pass HAVAL
}

\author{
Kazuhiro Suzuki $^{1}$, Kaoru Kurosawa ${ }^{2}$ \\ 1 Venture Business Laboratory, Ibaraki University, Hitachi, Ibaraki 316-8511, Japan \\ 2 Department of Computer and Information Sciences, Ibaraki University, Hitachi, \\ Ibaraki 316-8511, Japan
}

\begin{abstract}
The hash function HAVAL is an Australian extension of well known Merkle-Damgård hash functions such as MD4 and MD5. It has three variants, 3-, 4- and 5-pass HAVAL. On 3-pass HAVAL, the best known attack finds a collision pair with $2^{7}$ computations of the compression function. To find $k$ collision pairs, it requires $2^{7} k$ computations. In this paper, we present a better collision attack on 3-pass HAVAL, which can find $k$ collision pairs with only $2 k+33$ computations. Further, our message differential is different from the previous ones. (It is important to find collisions for different message differentials.)
\end{abstract}

Key words: hash function, HAVAL, collision, differential attack

\section{Introduction}

The hash function HAVAL was proposed by Zheng, Pieprzyk, and Seberry at Auscrypt '92 [8]. It is an Australian extension of well known Merkle-Damgård hash functions such as MD4 and MD5. ${ }^{3}$ HAVAL has three variants, 3-, 4- and 5-pass HAVAL, which means that the compression function has 96, 128, and 160 rounds, respectively. The compression function $H$ of HAVAL takes a 256-bit initial value and a 1024-bit message $M=\left(m_{0}, \ldots, m_{31}\right)$ as input, and produces 256-bit hash value as output, where each $m_{i}$ is a 32-bit word.

On 3-pass HAVAL, Rompay, et al. [2] presented a collision attack that requires $2^{29}$ computations of the compression function. Their attack can find a one-block (1024-bit) collision pair $M=\left(m_{0}, \ldots, m_{31}\right)$ and $M^{\prime}=\left(m_{0}^{\prime}, \ldots, m_{31}^{\prime}\right)$ with the differential

$$
\Delta m_{28}=2^{0}=1 \text { and } \Delta m_{i}=0 \text { for the other } i .
$$

X.Y.Wang et al. [4] showed a much better collision attack with $2^{7}$ computations of the compression function. Their attack can find a one-block collision pair $M=\left(m_{0}, \ldots, m_{31}\right)$ and $M^{\prime}=\left(m_{0}^{\prime}, \ldots, m_{31}^{\prime}\right)$ with the differential

$$
\Delta m_{0}=2^{10}, \Delta m_{11}=2^{31}, \Delta m_{18}=2^{3} \text {, and } \Delta m_{i}=0 \text { for the other } i .
$$

\footnotetext{
3 The newest version is HAVAL 1.1. We can download the program source code at the website [1]. The difference between the first version and HAVAL 1.1 is only the order of initial values and the other constant values.
} 
To find $k$ collision pairs of 3-pass HAVAL, the best known attack [4] requires $2^{7} k$ computations.

In this paper, we present a better collision attack on 3-pass HAVAL which can find $k$ collision pairs with only $2 k+33$ computations. Further, our message differential is different from the previous ones. ${ }^{4}$ (It is important to find collisions for different message differentials.)

The previous attacks $[2,4]$ are one-block collision attacks (i.e. a collision pair is a pair of 1024-bit message block). On the other hand, our attack is a two-block collision attack which can find a two-block (2048-bit) collision pair $M_{0} \| M_{1}=$ $\left(m_{0,0}, \ldots, m_{0,31}, m_{1,0}, \ldots, m_{1,31}\right)$ and $M_{0}^{\prime} \| M_{1}^{\prime}=\left(m_{0,0}^{\prime}, \ldots, m_{0,31}^{\prime}, m_{1,0}^{\prime}, \ldots\right.$, $\left.m_{1,31}^{\prime}\right)$ with the differential

$$
\Delta m_{j, i}=m_{j, i}^{\prime}-m_{j, i} \bmod 2^{32}= \begin{cases}2^{31} & \text { if } i=5 \\ 0 & \text { otherwise }\end{cases}
$$

In our attack, we first find a near-collision pair $\left(M_{0}, M_{0}^{\prime}\right)$ such that $H\left(M_{0}\right)$ and $H\left(M_{0}^{\prime}\right)$ are almost the same. We then find many full collision pairs $\left(M_{0} \| M_{1}\right.$, $\left.M_{0}^{\prime} \| M_{1}^{\prime}\right)$ by using the freedom of $\left(M_{1}, M_{1}^{\prime}\right)$. Theoretically, our near-collision pair can be found by about 33 computations of the compression function. Once a near collision pair is found, a full collision pair can be found with probability $1 / 2$. Hence we can find $k$ collision pairs with $2 k+33$ computations. (See Table 1.)

\begin{tabular}{|c|c|c|c|}
\hline & Rompay, et al. [2] & X.Y.Wang et al. [7] & Proposed \\
\hline$\Delta m_{i}$ & $\Delta m_{28}=2^{0}=1$ & $\begin{array}{l}\Delta m_{0}=2^{10} \\
\Delta m_{11}=2^{31} \\
\Delta m_{18}=2^{3}\end{array}$ & $\begin{aligned} \Delta m_{0,5} & =2^{31} \\
\Delta m_{1,5} & =2^{31}\end{aligned}$ \\
\hline complexity for first collision & $2^{29}$ & $2^{7}$ & $2+33$ \\
\hline complexity for $k$ collision pairs & $2^{29} k$ & $2^{7} k$ & $2 k+33$ \\
\hline message length & 1024 bits & 1024 bits & 2048 bits \\
\hline
\end{tabular}

Table 1. Collision attacks on 3-pass HAVAL

In our personal computer simulation:

1. We found 15147 near-collision pairs by 500000 trials, which agrees with our theoretical estimate because $500000 / 15147=33.0098 \cdots$.

2. From a single near-collision pair, we found 249630 full collision pairs by 500000 trials, which also agrees with our theoretical complexity because $500000 / 249630 \approx 2$.

\footnotetext{
${ }^{4}$ Our differential is used in an attack on 4-pass HAVAL by H.Yu et al. [7], but it is new for 3-pass HAVAL.
} 
It took about one minute for the first 500000 trials. It also took about one minute for the next 500000 trials.

\section{(Related wroks:)}

- Modular differential attack was presented in 1997 by X.Y.Wang [3] and formalized in Eurocrypt '05 [5,6]. They showed that it is very powerful to break MD4, MD5, SHA-0, SHA-1 and HAVAL. Our attack is also based on the modular differential approach.

- On 4-pass HAVAL, H.Yu et al. [7] showed two two-block collision attacks that require $2^{43}$ and $2^{36}$ computations of 4-pass HAVAL, respectively. On 5-pass HAVAL, the H.Yu et al. [7] showed a one-block collision attack with $2^{123}$ computations of the compression function.

This paper is organized as follows. In Section 2, we provide a simple description of 3-pass HAVAL. In Section 3, we give an outline of our attack. In Section 4 , we present the algorithm of our attack, and calculate the complexity. In Section 5, we report on our computational experiment and a collision example. In Section 6, we conclude this paper. In this paper, almost Tables and Figures are in the Appendix.

\section{3-Pass HAVAL}

HAVAL consists of three phases: (1) message padding phase, (2) main hashing phase and (3) optional compression phase.

\subsection{Message Padding Phase}

HAVAL pads an input message by appending some bit string so that its bitlength becomes a multiple of 1024 .

\subsection{Main Hashing Phase}

HAVAL is a Merkle-Damgård hash function based on a compression function $H$ as follows. Let $M_{0} \| M_{1}|| \cdots|| M_{t}$ be the padded message, where $\left|M_{i}\right|=1024$. Then for $i=0, \cdots, t$, compute

$$
I V_{i+1}=H\left(I V_{i}, M_{i}\right)
$$

where $\left|I V_{i}\right|=256$ and $I V_{0}=(a, b, c, d, e, f, g, h)$ is the initial value such that

$$
\begin{aligned}
& a=0 x 243 f 6 a 88, b=0 x 85 a 308 d 3, c=0 x 13198 a 2 e, d=0 x 03707344 \\
& e=0 x a 4093822, f=0 x 299 f 31 d 0, g=0 x 082 e f a 98, h=0 x e c 4 e 6 c 89 .
\end{aligned}
$$

The hashed value is given by $I V_{t+1}$.

$H$ is described as follows. First define three functions as follows. 


$$
\begin{aligned}
F_{1}\left(x_{0}, x_{1}, x_{2}, x_{3}, x_{4}, x_{5}, x_{6}\right)=\left(x_{2} \bullet x_{3}\right) & \oplus\left(x_{2} \bullet x_{4}\right) \oplus x_{4} \oplus\left(x_{0} \bullet x_{6}\right) \oplus\left(x_{1} \bullet x_{5}\right), \\
F_{2}\left(x_{0}, x_{1}, x_{2}, x_{3}, x_{4}, x_{5}, x_{6}\right)=\left(x_{0} \bullet x_{2}\right) & \oplus\left(x_{1} \bullet x_{2}\right) \oplus\left(x_{1} \bullet x_{3}\right) \oplus\left(x_{0} \bullet x_{3} \bullet x_{5}\right) \\
& \oplus\left(x_{1} \bullet x_{2} \bullet x_{5}\right) \oplus\left(x_{3} \bullet x_{5}\right) \oplus\left(x_{4} \bullet x_{5}\right) \\
& \oplus x_{6} \oplus\left(x_{5} \bullet x_{6}\right), \\
F_{3}\left(x_{0}, x_{1}, x_{2}, x_{3}, x_{4}, x_{5}, x_{6}\right)=x_{0} \quad & \oplus\left(x_{0} \bullet x_{3}\right) \oplus\left(x_{1} \bullet x_{4}\right) \oplus\left(x_{2} \bullet x_{5}\right) \\
& \oplus\left(x_{3} \bullet x_{4} \bullet x_{5}\right) \oplus\left(x_{3} \bullet x_{6}\right),
\end{aligned}
$$

where $x_{i}$ is a 32-bit word, $x_{i} \bullet x_{j}$ is the bit-wise multiplication of $x_{i}$ and $x_{j}$, and $x_{i} \oplus x_{j}$ is the bit-wise modulo 2 addition.

$H$ next runs the following algorithm $\tilde{H}$ on input

$$
\begin{aligned}
I V & =\left(a_{0}, b_{0}, c_{0}, d_{0}, e_{0}, f_{0}, g_{0}, h_{0}\right), \\
M & =\left(m_{0}, m_{1}, \ldots, m_{31}\right)
\end{aligned}
$$

where each of $a_{0}, \ldots, h_{0}$ and $m_{i}$ is a 32-bit word.

$\operatorname{For}(i=0$ to 95$)\{$

$$
\begin{aligned}
j & :=\lfloor i / 32\rfloor+1 \\
p_{i} & :=F_{j}\left(a_{i}, b_{i}, c_{i}, d_{i}, e_{i}, f_{i}, g_{i}\right) \\
a_{i+1} & :=\left(p_{i} \gg 7\right)+\left(h_{i} \gg 11\right)+m_{\text {ord }(i)}+k_{i} \bmod 2^{32} \\
b_{i+1} & :=a_{i}, c_{i+1}:=b_{i}, d_{i+1}:=c_{i}, e_{i+1}:=d_{i}, \\
f_{i+1} & :=e_{i}, g_{i+1}:=f_{i}, h_{i+1}:=g_{i}
\end{aligned}
$$

\}

where $x \gg s$ denotes the $s$-bit right rotation of $x,+$ denotes the modulo $2^{32}$ addition, and the word processing orders $\operatorname{ord}(i)$ and the constant values $k_{i}$ are given in Table 3 . Note that $\tilde{H}$ consists of 96 rounds, 0-round through 95-round.

Finally, $H$ outputs the following 256-bit value

$$
\begin{aligned}
& I V+\tilde{H}(I V, M) \\
& =\left(a_{0}+a_{96}, b_{0}+b_{96}, c_{0}+c_{96}, d_{0}+d_{96}, e_{0}+e_{96}, f_{0}+f_{96}, g_{0}+g_{96}, h_{0}+h_{96}\right) .
\end{aligned}
$$

Figure 1 is an outline sketch of $H$. In the Appendix, we provide more detailed sketches for each round. (See Figure 2, 3, and 4.)

\subsection{Optional Compression Phase}

HAVAL supports hash-sizes of 128, 160, 192, 224 and 256 bits. The main algorithm computes 256-bit hash-values, and the other sizes are obtained by postprocessing the 256-bit hash-value. 


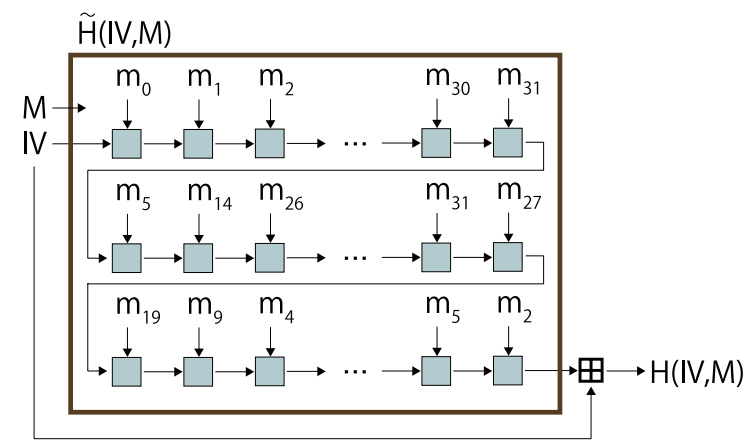

Fig. 1. The compression function $H$ of 3-pass HAVAL.

\section{Outline of our Attack}

\subsection{Notation}

For $H(I V, M), a_{i}, b_{i}, \cdots$ denote the local values which appear in $i$-round. Similarly, for $H\left(I V, M^{\prime}\right), a_{i}^{\prime}, b_{i}^{\prime}, \cdots$ denote the local values which appear in $i$-round. We denote by $a a_{0}, b b_{0}, c c_{0}, d d_{0}, e e_{0}, f f_{0}, g g_{0}, h h_{0}$ the 8 words of $I V_{1}$. Define $\Delta a_{i}=a_{i}^{\prime}-a_{i} \bmod 2^{32}$, and so on.

We denote by $x_{i, j}$ the $j$-th bit of 32-bit word $x_{i}$.

- $x_{i}^{\prime}=x_{i}[j]$ means that $x_{i}^{\prime}$ is obtained by changing the $j$ th bit of $x_{i}$ from 0 to 1. That is, $x_{i}^{\prime}=x_{i}$ except for that $x_{i, j}=0$ and $x_{i, j}^{\prime}=1$.

- $x_{i}^{\prime}=x_{i}[-j]$ means that $x_{i}^{\prime}$ is obtained by changing the $j$ th bit of $x_{i}$ from 1 to 0 . That is, $x_{i}^{\prime}=x_{i}$ except for that $x_{i, j}=1$ and $x_{i, j}^{\prime}=0$.

- $x_{i}^{\prime}=x_{i}[ \pm j]$ means that $x_{i, j} \neq x_{i, j}^{\prime}$.

- For example, $a_{14}[-21,22]$ is the value obtained by modifying the 21-th and 22-th bit of $a_{14}$ from 1 to 0 and 0 to 1 , respectively.

\subsection{Attack}

We show an efficient method to find a two-block (2048-bit) collision pair

$$
\begin{aligned}
& M_{0} \| M_{1}=\left(m_{0,0}, \ldots, m_{0,31}, m_{1,0}, \ldots, m_{1,31}\right) \\
& M_{0}^{\prime} \| M_{1}^{\prime}=\left(m_{0,0}^{\prime}, \ldots, m_{0,31}^{\prime}, m_{1,0}^{\prime}, \ldots, m_{1,31}^{\prime}\right) .
\end{aligned}
$$

The proposed method first finds a near collision pair $\left(M_{0}, M_{0}^{\prime}\right)$ such that

$$
\Delta I V_{1}=I V_{1}^{\prime}-I V_{1}=\left(0,2^{31}, 0,0,0,0,0,0\right)
$$

We next find a pair $\left(M_{1}, M_{1}^{\prime}\right)$ such that

$$
\begin{aligned}
\Delta A & =\tilde{H}\left(I V_{1}^{\prime}, M_{1}^{\prime}\right)-\tilde{H}\left(I V_{1}, M_{1}\right) \\
& =\left(0,2^{31}, 0,0,0,0,0,0\right) \text { or }\left(0,-2^{31}, 0,0,0,0,0,0\right)
\end{aligned}
$$


Then it holds that

$$
H\left(I V_{1}^{\prime}, M_{1}^{\prime}\right)=I V_{1}^{\prime}+\tilde{H}\left(I V_{1}^{\prime}, M_{1}^{\prime}\right)=I V_{1}+\tilde{H}\left(I V_{1}, M_{1}\right)=H\left(I V_{1}, M_{1}\right)
$$

That is,

$$
\Delta I V_{0}=0 \stackrel{\left(M_{0}, M_{0}^{\prime}\right)}{\longrightarrow} \Delta I V_{1} \stackrel{\left(M_{1}, M_{1}^{\prime}\right)}{\longrightarrow} \Delta A \rightarrow \Delta H=0
$$

Therefore, $\left(M_{0}\left\|M_{1}, M_{0}^{\prime}\right\| M_{1}^{\prime}\right)$ is a collision pair. ${ }^{5}$

We use a message differential such that

$$
\Delta m_{j, i}=m_{j, i}^{\prime}-m_{j, i} \bmod 2^{32}= \begin{cases}2^{31} & \text { if } i=5 \\ 0 & \text { otherwise }\end{cases}
$$

for $j=0$ and $1 .{ }^{6}$ That is,

$$
\begin{aligned}
& \Delta M_{0}=\left(0,0,0,0,0,2^{31}, 0, \cdots, 0\right) \\
& \Delta M_{1}=\left(0,0,0,0,0,2^{31}, 0, \cdots, 0\right)
\end{aligned}
$$

Note that $m_{5}$ is the input to the 5-, 32-, and 94-round in each block because

$$
5=\operatorname{ord}(5)=\operatorname{ord}(32)=\operatorname{ord}(94)
$$

from Table 3. Now we will find the first block pair $\left(M_{0}, M_{0}^{\prime}\right)$ that causes a local collision at the 32 -round. Then $\left(M_{0}, M_{0}^{\prime}\right)$ is automatically a near collision pair just after 94 -round with difference $\left(0, \pm 2^{31}, 0,0,0,0,0,0\right)$. This can be seen from the following table.

\begin{tabular}{|c|c|c|c|c|c|c|c|c|}
\hline round & 0 & $\cdots$ & 5 & $\cdots$ & 32 & $\cdots$ & 94 & 95 \\
\hline$\Delta m_{i}$ & $\Delta m_{0}=0$ & $\cdots$ & $\Delta m_{5}=2^{31}$ & $\cdots$ & $\Delta m_{5}=2^{31}$ & $\cdots$ & $\Delta m_{5}=2^{31}$ & $\cdots$ \\
\hline$\tilde{H}(I V, M)$ & & & & & collision & & near collision & near collision \\
\hline
\end{tabular}

Similarly we will find the second block pair $\left(M_{1}, M_{1}^{\prime}\right)$ which satisfy eq.(2), where $I V_{1}=H\left(I V_{0}, M_{0}\right)$ and $I V_{1}^{\prime}=H\left(I V_{0}, M_{0}^{\prime}\right)$. Then $M_{0} \| M_{0}^{\prime}$ and $M_{1} \| M_{1}^{\prime}$ are a full collision pair from eq.(3).

We present the (so called) differential path in Table 4 and Table $5 .{ }^{7}$ In these Table, for example,

$$
\begin{array}{|c|c|c|l|l|}
\hline \text { round } i & m_{i}^{\prime} & \Delta a_{i+1} & \text { Outputs } a_{i}^{\prime}, b_{i}^{\prime}, c_{i}^{\prime}, d_{i}^{\prime}, e_{i}^{\prime}, f_{i}^{\prime}, g_{i}^{\prime}, h_{i}^{\prime} \\
\hline 6 & m_{6} & 0 & a_{7}, a_{6}[32], a_{5}, a_{4}, a_{3}, a_{2}, a_{1}, a_{0} \\
\hline
\end{array}
$$

means that we want the outputs $\left(a_{7}^{\prime}, b_{7}^{\prime}, c_{7}^{\prime}, d_{7}^{\prime}, e_{7}^{\prime}, f_{7}^{\prime}, g_{7}^{\prime}, h_{7}^{\prime}\right)$ in the 6-round of $H\left(I V^{\prime}, M^{\prime}\right)$ to be $\left(a_{7}, b_{7}[32], c_{7}, d_{7}, e_{7}, f_{7}, g_{7}, h_{7}\right)$. Note that $\left(a_{7}, b_{7}, c_{7}, d_{7}, e_{7}\right.$, $\left.f_{7}, g_{7}, h_{7}\right)=\left(a_{7}, a_{6}, a_{5}, a_{4}, a_{3}, a_{2}, a_{1}, a_{0}\right)$. We can find a full collision if all the conditions of these tables are satisfied.

\footnotetext{
$\overline{5}$ The operation + is the word-wise modular $2^{32}$ addition.

6 This differential was used for 4-pass HAVAL [7]. Its complexity is $2^{43}$.

7 Table 4 was given in [7]. We constructed Table 5 .
} 


\subsection{Sufficient Conditions}

In Table 6, 7, and 8 , we present sufficient conditions for the differential path to hold. If Table 6,7 , and 8 are satisfied, then Table 4 and 5 are satisfied. As an example, we prove that the conditions for 5 - and 6-round given in Table 6 guarantee that the conditions for 0 - to 6 -round shown in Table 4 . The other conditions are derived similarly.

Since $\Delta m_{0}=\cdots=\Delta m_{4}=0$, the differential path of 0- to 4-round hold. In 5-round of $H\left(I V_{0}^{\prime}, M_{0}^{\prime}\right)$, if the sufficient condition of 5-round in Table 6, that is, $a_{6,32}=0$, then $a_{6,32}^{\prime}=1$ and $a_{6, i}^{\prime}=a_{6, i}$ for $i \neq 32$ (i.e. $a_{6}^{\prime}=a_{6}[32]$ ), because

$$
\begin{aligned}
a_{6} & =\left(p_{5} \gg 7\right)+\left(h_{5} \gg 11\right)+m_{\text {ord }(5)}+k_{5} \\
a_{6}^{\prime} & =\left(p_{5}^{\prime} \gg 7\right)+\left(h_{5}^{\prime} \gg 11\right)+m_{\text {ord }(5)}^{\prime}+k_{5} \\
& =\left(p_{5} \gg 7\right)+\left(h_{5} \gg 11\right)+\left(m_{\text {ord }(5)}+2^{31}\right)+k_{5} \\
& =\left(p_{5} \gg 7\right)+\left(h_{5} \gg 11\right)+m_{\text {ord }(5)}+k_{5}+2^{31} \\
& =a_{6}+2^{31} .
\end{aligned}
$$

In 6-round of $H\left(I V_{0}^{\prime}, M_{0}^{\prime}\right)$, if the sufficient condition of 5-round in Table 6, that is, $a_{0,32}=0$ then, since

$$
\begin{aligned}
p_{6} & =F_{1}\left(a_{6}, b_{6}, c_{6}, d_{6}, e_{6}, f_{6}, g_{6}\right) \\
& =F_{1}\left(a_{6}, a_{5}, a_{4}, a_{3}, a_{2}, a_{1}, a_{0}\right) \\
& =\left(a_{4} \bullet a_{3}\right) \oplus\left(a_{4} \bullet a_{2}\right) \oplus a_{2} \oplus\left(a_{6} \bullet a_{0}\right) \oplus\left(a_{5} \bullet a_{1}\right),
\end{aligned}
$$

we have

$$
\begin{aligned}
p_{6,32} & =\left(a_{4,32} \bullet a_{3,32}\right) \oplus\left(a_{4,32} \bullet a_{2,32}\right) \oplus a_{2,32} \oplus\left(a_{6,32} \bullet a_{0,32}\right) \oplus\left(a_{5,32} \bullet a_{1,32}\right), \\
& =\left(a_{4,32} \bullet a_{3,32}\right) \oplus\left(a_{4,32} \bullet a_{2,32}\right) \oplus a_{2,32} \oplus(0 \bullet 0) \oplus\left(a_{5,32} \bullet a_{1,32}\right), \\
& =\left(a_{4,32} \bullet a_{3,32}\right) \oplus\left(a_{4,32} \bullet a_{2,32}\right) \oplus a_{2,32} \oplus 0 \oplus\left(a_{5,32} \bullet a_{1,32}\right), \\
p_{6,32}^{\prime} & =\left(a_{4,32}^{\prime} \bullet a_{3,32}^{\prime}\right) \oplus\left(a_{4,32}^{\prime} \bullet a_{2,32}^{\prime}\right) \oplus a_{2,32}^{\prime} \oplus\left(a_{6,32}^{\prime} \bullet a_{0,32}^{\prime}\right) \oplus\left(a_{5,32}^{\prime} \bullet a_{1,32}^{\prime}\right), \\
& =\left(a_{4,32}^{\prime} \bullet a_{3,32}^{\prime}\right) \oplus\left(a_{4,32}^{\prime} \bullet a_{2,32}^{\prime}\right) \oplus a_{2,32}^{\prime} \oplus(1 \bullet 0) \oplus\left(a_{5,32}^{\prime} \bullet a_{1,32}^{\prime}\right), \\
& =\left(a_{4,32}^{\prime} \bullet a_{3,32}^{\prime}\right) \oplus\left(a_{4,32}^{\prime} \bullet a_{2,32}^{\prime}\right) \oplus a_{2,32}^{\prime} \oplus 0 \oplus\left(a_{5,32}^{\prime} \bullet a_{1,32}^{\prime}\right),
\end{aligned}
$$

Hence, $p_{6}^{\prime}=p_{6}$. Therefore $a_{7}^{\prime}=a_{7}$. The above equations for $p_{6,32}$ and $p_{6,32}^{\prime}$ are clear by Figure 2 .

\section{Details}

In this section, we present the details of our algorithm, and calculate the complexity to find a collision pair.

\subsection{Our Algorithm}

We observe that from $a_{1}, \cdots, a_{32}, M_{0}=\left(m_{0}, \ldots, m_{31}\right)$ is uniquley determined from eq.(1), and $a_{33}, \cdots, a_{96}$ are also uniquely determined. Now in Table 6 , all the rows except the last three rows specify the conditions on $a_{1}, \cdots, a_{32}$. Hence: 
1. We choose $a_{1}, \cdots, a_{32}$ which satisfy these conditions randomly.

2. We compute $M_{0}=\left(m_{0}, \ldots, m_{31}\right)$ from eq.(1). ${ }^{8}$

3. If the last three rows are also satisfied, then we have done.

Next for given $M_{0}$, we apply the same strategy to find $M_{1}$.

1. We choose $a_{1}, \cdots, a_{32}$ which satisfy the conditions of Table 7 and Table 8 randomly.

2. We compute $M_{1}$ from eq.(1).

3. If the last row of Table 8 is also satisfied, then we have done.

\section{Finding $M_{0}$ :}

1. Randomly select $a_{1}, \ldots, a_{32}$ that satisfy the sufficient conditions for 0-31 rounds.

2. For $i=0$ to 31 , $b_{i+1}:=a_{i}, c_{i+1}:=b_{i}, d_{i+1}:=c_{i}, e_{i+1}:=d_{i}$, $f_{i+1}:=e_{i}, g_{i+1}:=f_{i}, h_{i+1}:=g_{i}$.

3. Calculate $p_{0}, \ldots, p_{31}$ of the algorithm of $H$ in Section 2 and $m_{0}, \ldots, m_{31}$ as follows, $p_{i}:=F_{1}\left(a_{i}, b_{i}, c_{i}, d_{i}, e_{i}, f_{i}, g_{i}\right)$, $m_{i}:=a_{i+1}-\left(p_{i} \gg 7\right)-\left(h_{i} \gg 11\right)-k_{i} \bmod 2^{32}$.

4. Execute 32- to 95-round of the compression function.

5. If $a_{95,32}=1, a_{92,32}=1, b b_{0,32}=0, f f_{0,32}=0$, $c c_{0,32}=d d_{0,32}$, and $a a_{0,32}=0$, then fix $M_{0}=\left(m_{0}, \ldots, m_{31}\right)$.

\section{Finding $M_{1}$ :}

6. Randomly select $a_{1}, \ldots, a_{32}$ that satisfy the sufficient conditions for 0-31 rounds.

7. For $i=0$ to 31 , $b_{i+1}:=a_{i}, c_{i+1}:=b_{i}, d_{i+1}:=c_{i}, e_{i+1}:=d_{i}$, $f_{i+1}:=e_{i}, g_{i+1}:=f_{i}, h_{i+1}:=g_{i}$.

8. Calculate $p_{0}, \ldots, p_{31}$ of the algorithm of $H$ in Section 2 and $m_{0}, \ldots, m_{31}$ as follows, $p_{i}:=F_{1}\left(a_{i}, b_{i}, c_{i}, d_{i}, e_{i}, f_{i}, g_{i}\right)$, $m_{i}:=a_{i+1}-\left(p_{i} \gg 7\right)-\left(h_{i} \gg 11\right)-k_{i} \bmod 2^{32}$.

9. Execute 32- to 95-round of the compression function.

10. If $a_{92,32}=1$, then output $\left(M_{0} \| M_{1}\right)$ and $\left(M_{0}^{\prime} \| M_{1}^{\prime}\right)$ as a collision pair.

\subsection{Success Probability}

Assume that

$$
\operatorname{Pr}\left[x_{i, j}=0\right]=\operatorname{Pr}\left[x_{i, j}=1\right]=1 / 2
$$

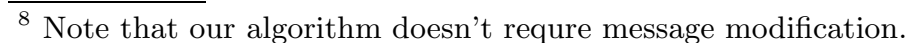


for any word $x_{i}$. We can find $M_{0}$ if the last three condition are satisfied in Table 6 . Thereore, the success probability $P$ of finding $M_{0}$ is given by

$$
\begin{aligned}
P & =\operatorname{Pr}\left[a_{95,32}=1, a_{92,32}=1, a a_{0,32}=0, b b_{0,32}=0, c c_{0,32}=d d_{0,32}, f f_{0,32}=0,\right. \\
& =1 / 2^{5} \times \operatorname{Pr}\left[b b_{0,32}=0\right]
\end{aligned}
$$

For $b b_{0}$, note that

$$
b b_{0}=b_{0}+b_{96}=b+a_{95}=(10000101 \cdots)+(1 ? ? ? ? ? ? ? \cdots) .
$$

Thus, if $a_{95,31}, a_{95,30}, a_{95,29}$, or $a_{95,28}$ is 0 , or $a_{95,31}=a_{95,30}=a_{95,29}=a_{95,28}=1$ and $a_{95,27}=a_{95,26}=0$, then $b b_{0,32}=0$. Hence

$$
\begin{aligned}
& \operatorname{Pr}\left[b b_{0,32}=0\right] \\
\geq & \operatorname{Pr}\left[a_{95,31}=0, a_{95,30}=0, a_{95,29}=0, \text { or } a_{95,28}=0\right] \\
& +\operatorname{Pr}\left[a_{95,31}=a_{95,30}=a_{95,29}=a_{95,28}=1 \text { and } a_{95,27}=a_{95,26}=0\right] \\
= & \left(1-\operatorname{Pr}\left[a_{95,31}=1, a_{95,30}=1, a_{95,29}=1, \text { and } a_{95,28}=1\right]\right)+1 / 2^{6} \\
= & \left(1-1 / 2^{4}\right)+1 / 64=15 / 16+1 / 64=61 / 64 .
\end{aligned}
$$

Therefore

$$
P \geq 1 / 2^{5} \times 61 / 64=61 / 2^{11} \approx 1 / 33
$$

Next suppose that the above $M_{0}$ is given. Then we can find $M_{1}$ if the last row of Table 8 is satisfied. Thereore, the success probability of finding $M_{1}$ is given by

$$
\operatorname{Pr}\left[a_{92,32}=1\right]=1 / 2 .
$$

\subsection{How to Find Many Collisions}

We can find many collision pairs from fixed $\left(M_{0}, M_{0}^{\prime}\right)$ by running the algorithm "Finding $M_{1}$ " many times. In this method, the complexity of finding $k$ collision pairs is $2 k+33$.

\section{Computational experiment}

We implemented our attack by a personal computer. First we found 15,147 desired $M_{0}$ s by running the algorithm "Finding $M_{0}$ " 500,000 times. In this experiment, the success probability $15,147 / 500,000 \approx 1 / 33$. It coincides with our theoretical probability shown in Section 3.

Next for fixed $M_{0}$, we found 249,630 desired $M_{1}$ s by running the algorithm "Finding $M_{1}$ " 500, 000 times. ${ }^{9}$ In this experiment, the success probability $249,630 / 500,000 \approx 1 / 2$. It coincides with our theoretical probability shown in Section 3.

In total, we found 249,630 full collision pairs by running the algorithms "Finding $M_{0}$ " 39 times and "Finding $M_{1}$ " 500, 000 times.

Consequently, our experiment supports our claim that we can find $k$ collision pairs with $2 k+33$ computations of the compression functions. We illustrate one of the 249630 collision pairs in Table 2.

\footnotetext{
${ }^{9}$ It takes about one minute on our computer.
} 


\begin{tabular}{|c|c|}
\hline & $\begin{array}{l}70 d 0972 \mathrm{~b} 027 \mathrm{ff} 7322476468056892 \mathrm{~d} 906 \mathrm{feca} 6 \mathrm{a} 0 \mathrm{a} 6 \mathrm{~b} 4 \mathrm{ec} \text { fbc11aca d12586db } \\
\text { e7bae1 ca89b85f 2d5a3e0f 8b4557da } 8596 \mathrm{~d} 1 \mathrm{bb} 2 \mathrm{bf5e} 1 \mathrm{fd} \text { b5b7f669 } 9445 \mathrm{ea} 9 \\
3860 \mathrm{ec} 5 \mathrm{c} 746759 \text { bbce300c d0985871 5229b382 8dab9e3e f89f39d6 } 9179329 \mathrm{~b}\end{array}$ \\
\hline${ }^{11}$ & 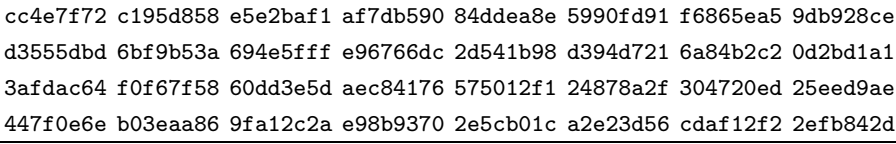 \\
\hline$u_{0}$ & $\begin{array}{l}\text { c7f10962 08cf4e0c ddfd60a8 597cbd0d b050440c a0 } \\
1270 d 0972 b 027 f f 7322476468056892 d 906 f e c a 6 \text { a0 } \\
\text { f7e7bae1 ca89b85f 2d5a3e0f } 8 \text { b4557da } 8596 d 1 b b 2 b \\
343860 e c 5 c 746759 \text { bbce300c d0985871 5229b382 8d }\end{array}$ \\
\hline$M_{1}^{\prime}$ & $\begin{array}{l}\text { cc4e7f72 c195d858 e5e2baf1 af7db590 } 84 \text { ddea8e d990fd91 f6865ea5 9db928ce } \\
\text { d3555dbd 6bf9b53a 694e5fff e96766dc 2d541b98 d394d721 6a84b2c2 0d2bd1a1 } \\
\text { 3afdac64 f0f67f58 60dd3e5d aec84176 } 575012 f 124878 a 2 f \text { 304720ed 25eed9ae } \\
447 f 0 e 6 e \text { b03eaa86 9fa12c2a e98b9370 } 2 \text { e5cb01c a2e23d56 cdaf12f2 2efb842d }\end{array}$ \\
\hline & \\
\hline
\end{tabular}

Table 2. Collision example.

\section{Conclusion}

On 3-pass HAVAL, the best known attack finds a collision pair with $2^{7}$ computations of the compression function. To find $k$ collision pairs, it requires $2^{7} k$ computations.

In this paper, we presented a better collision attack on 3-pass HAVAL using modular differential method. It can find $k$ collision pairs with only $2 k+33$ computations. Further, our message differential is different from the previous ones. (It is important to find collision pairs for different message differentials.)

\section{References}

1. Calyptix Security Corporation http://labs.calyptix.com/haval.php.: HAVAL Version 1.1, 2003.

2. Rompay, Bart Van; Biryukov, Alex; Preneel, Bart; Vandewalle, Joos: Cryptanalysis of 3-Pass HAVAL, Asiacrypt 2003, LNCS 2894, pp.228-245, 2003.

3. Wang, Xiaoyun: The Collision attack on SHA-0, in Chinese, to appear on www.infosec.sdu.edu.cn, 1997.

4. Wang, Xiaoyun; Feng, Dengguo; Yu, Xiuyuan: An attack on hash function HAVAL128, Science in China Ser.F Information Sciences 2005, Vol.48, No.5, pp.545-556.

5. Wang, Xiaoyun; Lai, Xuejia; Feng, Dengguo; Chen, Hui; Yu, Xiuyuan: Cryptanalysis of the Hash Functions MD4 and RIPEMD, Eurocrypt '05, LNCS 3494, pp.1-18, 2005.

6. Wang, Xiaoyun; Yu, Hongbo: How to Break MD5 and Other Hash Functions, Eurocrypt '05, LNCS 3494, pp.19-35, 2005. 
7. Yu, Hongbo; Wang, Xiaoyun; Yun, Aaram; Park, Sangwoo: Cryptanalysis of the Full HAVAL with 4 and 5 Passes, FSE 2006, LNCS 4047, pp.89-110, 2006.

8. Zheng, Yuliang; Pieprzyk, Josef; Seberry, Jennifer: HAVAL - A One-Way Hashing Algorithm with Variable Length of Output (Extended Abstract), Auscrypt '92, LNCS 718, pp.83-104, 1993.

\section{Appendix : Tables and Figures}

\begin{tabular}{|c|c|}
\hline$i$ & $\operatorname{ord}(i)$ \\
\hline 0 to 31 & $\begin{array}{cccccccccccccccc}0 & 1 & 2 & 3 & 4 & 5 & 6 & 7 & 8 & 9 & 10 & 11 & 12 & 13 & 14 & 15 \\
16 & 17 & 18 & 19 & 20 & 21 & 22 & 23 & 24 & 25 & 26 & 27 & 28 & 29 & 30 & 31\end{array}$ \\
\hline 2 to 63 & $\begin{array}{ccccccccccccccccc}5 & 14 & 26 & 18 & 11 & 28 & 7 & 16 & 0 & 23 & 20 & 22 & 1 & 10 & 4 & 8 \\
30 & 3 & 21 & 9 & 17 & 24 & 29 & 6 & 19 & 12 & 15 & 13 & 2 & 25 & 31 & 27\end{array}$ \\
\hline 0470 & $\begin{array}{cccccccccccccccc}19 & 9 & 4 & 20 & 28 & 17 & 8 & 22 & 29 & 14 & 25 & 12 & 24 & 30 & 16 & 26 \\
31 & 15 & 7 & 3 & 1 & 0 & 18 & 27 & 13 & 6 & 21 & 10 & 23 & 11 & 5 & 2\end{array}$ \\
\hline
\end{tabular}

\begin{tabular}{|c|c|}
\hline$i$ & $k_{i}$ \\
\hline \begin{tabular}{|lll}
0 & to 31
\end{tabular} & 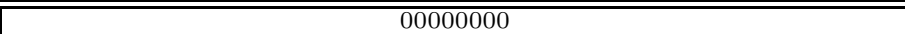 \\
\hline 32 to 63 & 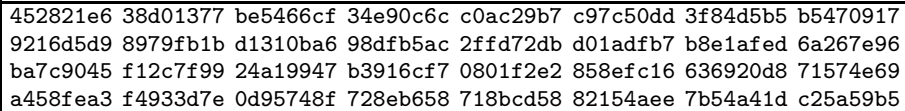 \\
\hline 64 to 95 & $\begin{array}{l}\text { 9c30d539 2af26013 c5d1b023 286085f0 ca417918 b8db38ef 8e79dcb0 603a180e } \\
\text { 6c9e0e8b b01e8a3e d71577c1 bd314b27 78af2fda 55605c60 e65525f3 aa55ab94 } \\
57489862 \text { 63e81440 55ca396a 2aab10b6 b4cc5c34 1141e8ce a15486af 7c72e993 } \\
\text { b3ee1411 636fbc2a 2ba9c55d 741831f6 ce5c3e16 9b87931e afd6ba33 6c24cf5c }\end{array}$ \\
\hline
\end{tabular}

Table 3. Word processing orders ord $(i)$ and constant values $k_{i}$ (hexadecimal numbers). 


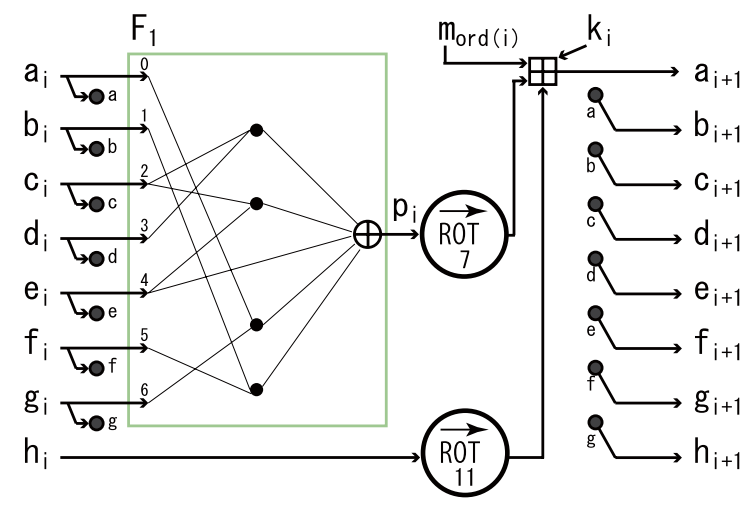

Fig. 2. $i$-round $(0 \leq i \leq 31)$ in the compression function.

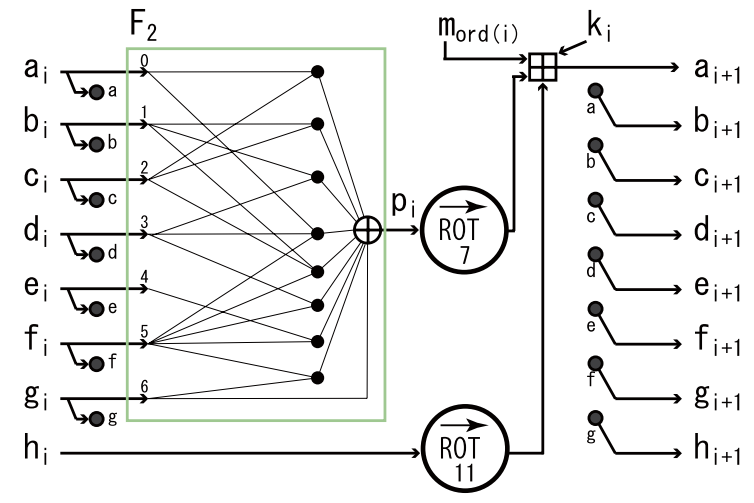

Fig. 3. $i$-round $(32 \leq i \leq 63)$ in the compression function.

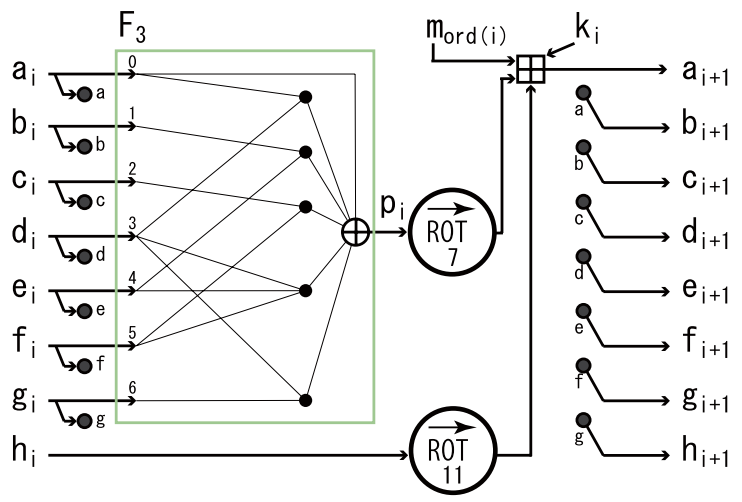

Fig. 4. $i$-round $(64 \leq i \leq 95)$ in the compression function. 


\begin{tabular}{|c|c|c|c|}
\hline round $i$ & $m_{i}^{\prime}$ & $\Delta a_{i+1}$ & Outputs $a_{i}^{\prime}, b_{i}^{\prime}, c_{i}^{\prime}, d_{i}^{\prime}, e_{i}^{\prime}, f_{i}^{\prime}, g_{i}^{\prime}, h_{i}^{\prime}$ \\
\hline$I V_{0}^{\prime}$ & & & $a_{0}, b_{0}, c_{0}, d_{0}, e_{0}, f_{0}, g_{0}, h_{0}$ \\
\hline 0 & $m_{0}$ & 0 & $a_{1}, a_{0}, b_{0}, c_{0}, d_{0}, e_{0}, f_{0}, g_{0}$ \\
\hline 1 & $m_{1}$ & 0 & $a_{2}, a_{1}, a_{0}, b_{0}, c_{0}, d_{0}, e_{0}, f_{0}$ \\
\hline 2 & $m_{2}$ & 0 & $a_{3}, a_{2}, a_{1}, a_{0}, b_{0}, c_{0}, d_{0}, e_{0}$ \\
\hline 3 & $m_{3}$ & 0 & $a_{4}, a_{3}, a_{2}, a_{1}, a_{0}, b_{0}, c_{0}, d_{0}$ \\
\hline 4 & $m_{4}$ & 0 & $a_{5}, a_{4}, a_{3}, a_{2}, a_{1}, a_{0}, b_{0}, c_{0}$ \\
\hline 5 & $m_{5}^{\prime}$ & $2^{31}$ & $a_{6}[32], a_{5}, a_{4}, a_{3}, a_{2}, a_{1}, a_{0}, b_{0}$ \\
\hline 6 & $m_{6}$ & 0 & $a_{7}, a_{6}[32], a_{5}, a_{4}, a_{3}, a_{2}, a_{1}, a_{0}$ \\
\hline 7 & $m_{7}$ & 0 & $a_{8}, a_{7}, a_{6}[32], a_{5}, a_{4}, a_{3}, a_{2}, a_{1}$ \\
\hline 8 & $m_{8}$ & 0 & $a_{9}, a_{8}, a_{7}, a_{6}[32], a_{5}, a_{4}, a_{3}, a_{2}$ \\
\hline 9 & $m_{9}$ & 0 & $a_{10}, a_{9}, a_{8}, a_{7}, a_{6}[32], a_{5}, a_{4}, a_{3}$ \\
\hline 10 & $m_{10}$ & 0 & $a_{11}, a_{10}, a_{9}, a_{8}, a_{7}, a_{6}[32], a_{5}, a_{4}$ \\
\hline 11 & $m_{11}$ & 0 & $a_{12}, a_{11}, a_{10}, a_{9}, a_{8}, a_{7}, a_{6}[32], a_{5}$ \\
\hline 12 & $m_{12}$ & 0 & $a_{13}, a_{12}, a_{11}, a_{10}, a_{9}, a_{8}, a_{7}, a_{6}[32]$ \\
\hline 13 & $m_{13}$ & $2^{20}$ & $a_{14}[-21,22], a_{13}, a_{12}, a_{11}, a_{10}, a_{9}, a_{8}, a_{7}$ \\
\hline 14 & $m_{14}$ & 0 & $a_{15}, a_{14}[-21,22], a_{13}, a_{12}, a_{11}, a_{10}, a_{9}, a_{8}$ \\
\hline 15 & $m_{15}$ & 0 & $a_{16}, a_{15}, a_{14}[-21,22], a_{13}, a_{12}, a_{11}, a_{10}, a_{9}$ \\
\hline 16 & $m_{16}$ & 0 & $a_{17}, a_{16}, a_{15}, a_{14}[-21,22], a_{13}, a_{12}, a_{11}, a_{10}$ \\
\hline 17 & $m_{17}$ & $-2^{14}$ & $a_{18}[15,16,17,-18], a_{17}, a_{16}, a_{15}, a_{14}[-21,22], a_{13}, a_{12}, a_{11}$ \\
\hline 18 & $m_{18}$ & 0 & $a_{19}, a_{18}[15,16,17,-18], a_{17}, a_{16}, a_{15}, a_{14}[-21,22], a_{13}, a_{12}$ \\
\hline 19 & $m_{19}$ & 0 & $a_{20}, a_{19}, a_{18}[15,16,17,-18], a_{17}, a_{16}, a_{15}, a_{14}[-21,22], a_{13}$ \\
\hline 20 & $m_{20}$ & 0 & $a_{21}, a_{20}, a_{19}, a_{18}[15,16,17,-18], a_{17}, a_{16}, a_{15}, a_{14}[-21,22]$ \\
\hline 21 & $m_{21}$ & 0 & $a_{22}, a_{21}, a_{20}, a_{19}, a_{18}[15,16,17,-18], a_{17}, a_{16}, a_{15}$ \\
\hline 22 & $m_{22}$ & 0 & $a_{23}, a_{22}, a_{21}, a_{20}, a_{19}, a_{18}[15,16,17,-18], a_{17}, a_{16}$ \\
\hline 23 & $m_{23}$ & 0 & $a_{24}, a_{23}, a_{22}, a_{21}, a_{20}, a_{19}, a_{18}[15,16,17,-18], a_{17}$ \\
\hline 24 & $m_{24}$ & $2^{10}$ & $a_{25}[11], a_{24}, a_{23}, a_{22}, a_{21}, a_{20}, a_{19}, a_{18}[15,16,17,-18]$ \\
\hline 25 & $m_{25}$ & 0 & $a_{26}, a_{25}[11], a_{24}, a_{23}, a_{22}, a_{21}, a_{20}, a_{19}$ \\
\hline 26 & $m_{26}$ & 0 & $a_{27}, a_{26}, a_{25}[11], a_{24}, a_{23}, a_{22}, a_{21}, a_{20}$ \\
\hline 27 & $m_{27}$ & 0 & $a_{28}, a_{27}, a_{26}, a_{25}[11], a_{24}, a_{23}, a_{22}, a_{21}$ \\
\hline 28 & $m_{28}$ & 0 & $a_{29}, a_{28}, a_{27}, a_{26}, a_{25}[11], a_{24}, a_{23}, a_{22}$ \\
\hline 29 & $m_{29}$ & 0 & $a_{30}, a_{29}, a_{28}, a_{27}, a_{26}, a_{25}[11], a_{24}, a_{23}$ \\
\hline 30 & $m_{30}$ & 0 & $a_{31}, a_{30}, a_{29}, a_{28}, a_{27}, a_{26}, a_{25}[11], a_{24}$ \\
\hline 31 & $m_{31}$ & 0 & $a_{32}, a_{31}, a_{30}, a_{29}, a_{28}, a_{27}, a_{26}, a_{25}[11]$ \\
\hline 32 & $m_{5}^{\prime}$ & 0 & $a_{33}, a_{32}, a_{31}, a_{30}, a_{29}, a_{28}, a_{27}, a_{26}$ \\
\hline$\cdots$ & $\cdots$ & $\cdots$ & $\cdots$ \\
\hline 94 & $m_{5}^{\prime}$ & $-2^{31}$ & $a_{95}[-32], a_{94}, a_{93}, a_{92}, a_{91}, a_{90}, a_{89}, a_{88}$ \\
\hline 95 & $m_{2}$ & 0 & $a_{96}, a_{95}[-32], a_{94}, a_{93}, a_{92}, a_{91}, a_{90}, a_{89}$ \\
\hline$I V_{1}^{\prime}$ & & & $a a_{0}, b b_{0}[32], c c_{0}, d d_{0}, e e_{0}, f f_{0}, g g_{0}, h h_{0}$ \\
\hline
\end{tabular}

Table 4. A differential path for $H\left(I V_{0}, M_{0}\right)$ and $H\left(I V_{0}, M_{0}^{\prime}\right)$. 


\begin{tabular}{|c|c|c|c|}
\hline round $i$ & $m_{i}^{\prime}$ & $\Delta a_{i+1}$ & Outputs $a_{i}^{\prime}, b_{i}^{\prime}, c_{i}^{\prime}, d_{i}^{\prime}, e_{i}^{\prime}, f_{i}^{\prime}, g_{i}^{\prime}, h_{i}^{\prime}$ \\
\hline$\overline{I I V_{1}^{\prime}}$ & & & $a_{0}, b_{0}[32], c_{0}, d_{0}, e_{0}, f_{0}, g_{0}, h_{0}$ \\
\hline 0 & $m_{0}$ & 0 & $a_{1}, a_{0}, b_{0}[32], c_{0}, d_{0}, e_{0}, f_{0}, g_{0}$ \\
\hline 1 & $m_{1}$ & 0 & $a_{2}, a_{1}, a_{0}, b_{0}[32], c_{0}, d_{0}, e_{0}, f_{0}$ \\
\hline 2 & $m_{2}$ & 0 & $a_{3}, a_{2}, a_{1}, a_{0}, b_{0}[32], c_{0}, d_{0}, e_{0}$ \\
\hline 3 & $m_{3}$ & 0 & $a_{4}, a_{3}, a_{2}, a_{1}, a_{0}, b_{0}[32], c_{0}, d_{0}$ \\
\hline 4 & $m_{4}$ & 0 & $a_{5}, a_{4}, a_{3}, a_{2}, a_{1}, a_{0}, b_{0}[32], c_{0}$ \\
\hline 5 & $m_{5}^{\prime}$ & $2^{31}$ & $a_{6}[32], a_{5}, a_{4}, a_{3}, a_{2}, a_{1}, a_{0}, b_{0}[32]$ \\
\hline 6 & $m_{6}$ & $2^{20}$ & $a_{7}[21], a_{6}[32], a_{5}, a_{4}, a_{3}, a_{2}, a_{1}, a_{0}$ \\
\hline 7 & $m_{7}$ & 0 & $a_{8}, a_{7}[21], a_{6}[32], a_{5}, a_{4}, a_{3}, a_{2}, a_{1}$ \\
\hline 8 & $m_{8}$ & $-2^{24}$ & $a_{9}[25,26,27,-28], a_{8}, a_{7}[21], a_{6}[32], a_{5}, a_{4}, a_{3}, a_{2}$ \\
\hline 9 & \begin{tabular}{|l|l|}
$m_{9}$ \\
\end{tabular} & 0 & $a_{10}, a_{9}[25,26,27,-28], a_{8}, a_{7}[21], a_{6}[32], a_{5}, a_{4}, a_{3}$ \\
\hline 10 & $m_{10}$ & $-2^{18}$ & $a_{11}[19,20,-21], a_{10}, a_{9}[25,26,27,-28], a_{8}, a_{7}[21], a_{6}[32], a_{5}, a_{4}$ \\
\hline 11 & $m_{11}$ & 0 & $a_{12}, a_{11}[19,20,-21], a_{10}, a_{9}[25,26,27,-28], a_{8}, a_{7}[21], a_{6}[32], a_{5}$ \\
\hline 12 & $m_{12}$ & $-2^{11}$ & $\begin{array}{l}a_{13}[12,13,14,15,16,-17], a_{12}, a_{11}[19,20,-21], a_{10} \\
a_{9}[25,26,27,-28], a_{8}, a_{7}[21], a_{6}[32] \\
\end{array}$ \\
\hline 13 & $m_{13}$ & $-2^{7}$ & $\begin{array}{l}a_{14}[-8], a_{13}[12,13,14,15,16,-17], a_{12}, a_{11}[19,20,-21], a_{10}, \\
a_{9}[25,26,27,-28], a_{8}, a_{7}[21]\end{array}$ \\
\hline 14 & $m_{14}$ & 0 & $\begin{array}{l}a_{15}, a_{14}[-8], a_{13}[12,13,14,15,16,-17], a_{12}, a_{11}[19,20,-21], a_{10} \\
a_{9}[25,26,27,-28], a_{8}\end{array}$ \\
\hline 15 & $m_{15}$ & 0 & $\begin{array}{l}a_{16}, a_{15}, a_{14}[-8], a_{13}[12,13,14,15,16,-17], a_{12}, a_{11}[19,20,-21] \\
a_{10}, a_{9}[25,26,27,-28]\end{array}$ \\
\hline 16 & $m_{16}$ & 0 & $\begin{array}{l}a_{17}, a_{16}, a_{15}, a_{14}[-8], a_{13}[12,13,14,15,16,-17], a_{12}, \\
a_{11}[19,20,-21], a_{10}\end{array}$ \\
\hline 17 & $m_{17}$ & 0 & $\begin{array}{l}a_{18}, a_{17}, a_{16}, a_{15}, a_{14}[-8], a_{13}[12,13,14,15,16,-17], a_{12}, \\
a_{11}[19,20,-21]\end{array}$ \\
\hline 18 & $m_{18}$ & 0 & $a_{19}, a_{18}, a_{17}, a_{16}, a_{15}, a_{14}[-8], a_{13}[12,13,14,15,16,-17], a_{12}$ \\
\hline 19 & $m_{19}$ & 0 & $a_{20}, a_{19}, a_{18}, a_{17}, a_{16}, a_{15}, a_{14}[-8], a_{13}[12,13,14,15,16,-17]$ \\
\hline 20 & $m_{20}$ & 0 & $a_{21}, a_{20}, a_{19}, a_{18}, a_{17}, a_{16}, a_{15}, a_{14}[-8]$ \\
\hline 21 & $m_{21}$ & $-2^{28}$ & $a_{22}[-29], a_{21}, a_{20}, a_{19}, a_{18}, a_{17}, a_{16}, a_{15}$ \\
\hline 22 & $m_{22}$ & $-2^{21}$ & $a_{23}[22,23,24,-25], a_{22}[-29], a_{21}, a_{20}, a_{19}, a_{18}, a_{17}, a_{16}$ \\
\hline 23 & $m_{23}$ & $-2^{14}$ & $\begin{array}{l}a_{24}[15,16,17,-18], a_{23}[22,23,24,-25], a_{22}[-29], a_{21}, a_{20}, a_{19}, \\
a_{18}, a_{17}\end{array}$ \\
\hline 24 & $m_{24}$ & $-2^{10}$ & $\begin{array}{l}a_{25}[-11], a_{24}[15,16,17,-18], a_{23}[22,23,24,-25], a_{22}[-29], a_{21}, \\
a_{20}, a_{19}, a_{18}\end{array}$ \\
\hline 25 & $m_{25}$ & 0 & $\begin{array}{l}a_{26}, a_{25}[-11], a_{24}[15,16,17,-18], a_{23}[22,23,24,-25], a_{22}[-29], \\
a_{21}, a_{20}, a_{19}\end{array}$ \\
\hline 26 & $m_{26}$ & 0 & $\begin{array}{l}a_{27}, a_{26}, a_{25}[-11], a_{24}[15,16,17,-18], a_{23}[22,23,24,-25], \\
a_{22}[-29], a_{21}, a_{20}\end{array}$ \\
\hline 27 & $m_{27}$ & 0 & $\begin{array}{l}a_{28}, a_{27}, a_{26}, a_{25}[-11], a_{24}[15,16,17,-18], a_{23}[22,23,24,-25], \\
a_{22}[-29], a_{21}\end{array}$ \\
\hline 28 & $m_{28}$ & 0 & $\begin{array}{l}a_{29}, a_{28}, a_{27}, a_{26}, a_{25}[-11], a_{24}[15,16,17,-18] \\
a_{23}[22,23,24,-25], a_{22}[-29]\end{array}$ \\
\hline 29 & $m_{29}$ & 0 & $\begin{array}{l}a_{30}, a_{29}, a_{28}, a_{27}, a_{26}, a_{25}[-11], a_{24}[15,16,17,-18] \\
a_{23}[22,23,24,-25]\end{array}$ \\
\hline 30 & $m_{30}$ & 0 & $a_{31}, a_{30}, a_{29}, a_{28}, a_{27}, a_{26}, a_{25}[-11], a_{24}[15,16,17,-18]$ \\
\hline 31 & $m_{31}$ & 0 & $a_{32}, a_{31}, a_{30}, a_{29}, a_{28}, a_{27}, a_{26}, a_{25}[-11]$ \\
\hline 32 & $m_{5}^{\prime}$ & 0 & $a_{33}, a_{32}, a_{31}, a_{30}, a_{29}, a_{28}, a_{27}, a_{26}$ \\
\hline$\cdots$ & \begin{tabular}{|l|l|}
$\cdots$ \\
\end{tabular} & $\cdots$ & $\cdots$ \\
\hline 94 & $m_{5}^{\prime}$ & $\pm 2^{31}$ & $a_{95}[ \pm 32], a_{94}, a_{93}, a_{92}, a_{91}, a_{90}, a_{89}, a_{88}$ \\
\hline 95 & $m_{2}$ & 0 & $a_{96}, a_{95}[ \pm 32], a_{94}, a_{93}, a_{92}, a_{91}, a_{90}, a_{89}$ \\
\hline$I V_{2}^{\prime}$ & & & $\begin{array}{l}a_{0}+a_{96}, b_{0}[32]+a_{95}[ \pm 32], c_{0}+a_{94}, d_{0}+a_{93}, e_{0}+a_{92}, f_{0}+a_{91}, g_{0}+ \\
a_{90}, h_{0}+a_{89} \quad \text { Full Collision !! }\end{array}$ \\
\hline
\end{tabular}

Table 5. A differential path for $H\left(I V_{1}, M_{1}\right)$ and $H\left(I V_{1}^{\prime}, M_{1}^{\prime}\right)$. 


\begin{tabular}{|c|c|}
\hline$\overline{\text { und }}$ & Sufficient conditions for each round \\
\hline$\overline{\overline{5}}$ & $a_{6,32}=0$ \\
\hline 6 & $a_{0,32}=0$ \\
\hline 7 & $a_{2,32}=0$ \\
\hline 8 & $a_{5,32}=a_{4,32}$ \\
\hline 9 & $a_{7,32}=0$ \\
\hline 10 & $a_{8,32}=1$ \\
\hline 11 & $a_{10,32}=0$ \\
\hline 12 & $a_{12,32}=0$ \\
\hline 13 & $a_{14,21}=1, a_{14,22}=0$ \\
\hline 14 & $a_{8,21}=0, a_{8,22}=0$ \\
\hline 15 & $a_{10,21}=0, a_{10,22}=0$ \\
\hline 16 & $a_{13,21}=a_{12,21}, a_{13,22}=a_{12,22}$ \\
\hline 17 & $\begin{array}{l}a_{18,15}=0, a_{18,16}=0, a_{18,17}=0, a_{18,18}=1, a_{15,21}=0, a_{15,22}= \\
1, a_{11,22}=0, a_{12,22}=1, a_{13,22}=1, a_{16,22}=1\end{array}$ \\
\hline 18 & $a_{12,15}=0, a_{12,16}=0, a_{12,17}=0, a_{12,18}=0, a_{16,21}=1, a_{16,22}=1$ \\
\hline 19 & $a_{14,15}=0, a_{14,16}=0, a_{14,17}=0, a_{14,18}=0, a_{18,21}=0, a_{18,22}=0$ \\
\hline 20 & $\begin{array}{l}a_{16,15}=a_{17,15}, a_{16,16}=a_{17,16}, a_{16,17}=a_{17,17}, a_{16,18}=a_{17,18} \\
a_{20,21}=0, a_{20,22}=0\end{array}$ \\
\hline 21 & $\begin{array}{l}a_{19,15}=0, a_{19,16}=0, a_{19,17}=1, a_{19,18}=0, a_{15,17}=1, a_{16,17}= \\
0, a_{17,17}=0, a_{21,17}=1\end{array}$ \\
\hline 22 & $a_{20,15}=1, a_{20,16}=1, a_{20,17}=1, a_{20,18}=1$ \\
\hline 23 & $a_{22,15}=0, a_{22,16}=0, a_{22,17}=0, a_{22,18}=0$ \\
\hline 24 & $a_{24,15}=0, a_{24,16}=0, a_{24,17}=0, a_{24,18}=1, a_{25,11}=0$ \\
\hline 25 & $a_{19,11}=1, a_{20,11}=0, a_{22,11}=0, a_{21,11}=0$ \\
\hline 26 & $a_{21,11}=0$ \\
\hline 27 & $a_{24,11}=a_{23,11}$ \\
\hline 28 & $a_{26,11}=0$ \\
\hline 29 & $a_{27,11}=1$ \\
\hline 30 & $a_{29,11}=0$ \\
\hline 31 & $a_{31,11}=0$ \\
\hline 94 & $a_{95,32}=1$ \\
\hline 95 & $a_{92,32}=1$ \\
\hline$\overline{I I V_{1}}$ & $a a_{0,32}=0, b b_{0,32}=0, c c_{0,32}=d d_{0,32}, f f_{0,32}=0$ \\
\hline
\end{tabular}

Table 6. Sufficient conditions on $a_{i}$ for the differential path in Table 4 . 


\begin{tabular}{|c|c|}
\hline & $\mathrm{d}$ \\
\hline 0 & $f_{0,32}=0$ \\
\hline 1 & $c_{0,32}=d_{0,32}$ \\
\hline 2 & $a_{0,32}=0$ \\
\hline 3 & $a_{1,32}=1$ \\
\hline 4 & $a_{3,32}=0$ \\
\hline 5 & $a_{5,32}=0, a_{6,32}=0$ \\
\hline 6 & $a_{7,21}=0, a_{0,32}=0$ \\
\hline 7 & $a_{1,21}=0, a_{2,32}=0$ \\
\hline 8 & $\begin{array}{l}a_{9,25}=0, a_{9,26}=0, a_{9,27}=0, a_{9,28}=1, a_{3,21}=0, a_{2,32}=0, a_{3,32}= \\
0, a_{4,32}=1, a_{5,32}=0\end{array}$ \\
\hline 9 & $a_{7,32}=0, a_{5,21}=a_{6,21}, a_{3,25}=0, a_{3,26}=0, a_{3,27}=0, a_{3,28}=0$ \\
\hline 10 & $\begin{array}{l}a_{11,19}=0, a_{11,20}=0, a_{11,21}=1, a_{8,32}=1, a_{8,21}=0, a_{5,25}=0, a_{5,26}= \\
1, a_{5,27}=0, a_{5,28}=0, a_{8,26}=0, a_{6,26}=1, a_{4,26}=0\end{array}$ \\
\hline 11 & $\begin{array}{l}a_{5,19}=0, a_{5,20}=0, a_{9,21}=a_{5,21}+1, a_{7,25}=a_{8,25}, a_{7,26}=a_{8,26}, a_{8,27}= \\
a_{7,27}, a_{7,28}=a_{8,28}, a_{10,32}=0\end{array}$ \\
\hline 12 & $\begin{array}{l}a_{13,12}=0, a_{13,13}=0, a_{13,14}=0, a_{13,15}=0, a_{13,16}=0, a_{13,17}=1, a_{12,32}= \\
0, a_{7,19}=1, a_{7,20}=0, a_{10,25}=0, a_{10,26}=0, a_{10,27}=0, a_{10,28}=0, a_{8,19}= \\
1, a_{10,19}=0, a_{6,19}=0\end{array}$ \\
\hline 13 & $\begin{array}{l}a_{14,8}=1, a_{7,12}=0, a_{7,13}=0, a_{7,14}=0, a_{7,15}=1, a_{7,16}=0, a_{7,17}= \\
0, a_{10,19}=a_{9,19}, a_{10,20}=a_{9,20}, a_{10,21}=a_{9,21}+1, a_{11,25}=1, a_{11,26}= \\
1, a_{11,27}=1, a_{11,28}=0, a_{7,28}=0, a_{8,28}=0, a_{8,15}=0, a_{9,15}=0, a_{10,15}= \\
1, a_{11,15}=1, a_{13,21}=1\end{array}$ \\
\hline 14 & $\begin{array}{l}a_{8,8}=0, a_{9,12}=0, a_{9,13}=0, a_{9,14}=0, a_{9,15}=0, a_{9,16}=0, a_{9,17}= \\
1, a_{12,19}=0, a_{12,20}=0, a_{12,21}=0, a_{13,25}=0, a_{13,26}=0, a_{13,27}=0, a_{13,28}= \\
0, a_{8,17}=0, a_{10,17}=0, a_{11,17}=0\end{array}$ \\
\hline 15 & $\begin{array}{l}a_{10,8}=0, a_{12,12}=a_{11,12}, a_{12,13}=a_{11,13}, a_{12,14}=a_{11,14}, a_{12,15}= \\
a_{11,15}, a_{12,16}=a_{11,16}, a_{12,17}=a_{11,17}, a_{13,19}=1, a_{13,20}=1, a_{13,21}= \\
1, a_{15,25}=0, a_{15,26}=0, a_{15,27}=0, a_{15,28}=0\end{array}$ \\
\hline 16 & $\begin{array}{l}a_{13,8}=a_{12,8}, a_{14,12}=0, a_{14,13}=0, a_{14,14}=0, a_{14,15}=0, a_{14,16}=0, a_{14,17}= \\
0, a_{15,19}=0, a_{15,20}=0, a_{15,21}=1, a_{10,21}=0, a_{12,21}=0, a_{13,21}=1, a_{14,21}= \\
1\end{array}$ \\
\hline 17 & $\begin{array}{l}a_{15,8}=0, a_{15,12}=1, a_{15,13}=1, a_{15,14}=1, a_{15,15}=1, a_{15,16}=1, a_{15,17}= \\
1, a_{17,19}=0, a_{17,20}=0, a_{17,21}=0\end{array}$ \\
\hline 18 & $\begin{array}{l}a_{16,8}=1, a_{17,12}=0, a_{17,13}=0, a_{17,14}=0, a_{17,15}=1, a_{17,16}=0, a_{17,17}= \\
0, a_{14,15}=0, a_{16,15}=0, a_{18,15}=0\end{array}$ \\
\hline 19 & $a_{18,8}=0, a_{19,12}=0, a_{19,13}=0, a_{19,14}=0, a_{19,15}=0, a_{19,16}=0, a_{19,17}=0$ \\
\hline 20 & $a_{16,8}=1, a_{18,8}=0, a_{20,8}=1$ \\
\hline
\end{tabular}

Table 7. Sufficient conditions on $a_{i}$ for 0-20 rounds of the differential path in Table 5 . 


\begin{tabular}{|c|l|}
\hline round $i$ & Sufficient conditions for each round \\
\hline \hline 21 & $a_{22,29}=1$ \\
\hline 22 & $\begin{array}{l}a_{23,22}=0, a_{23,23}=0, a_{23,24}=0, a_{23,25}=1, a_{16,19}=1, a_{17,29}=0, \\
a_{18,29}=0, a_{19,29}=0\end{array}$ \\
\hline 23 & $\begin{array}{l}a_{24,15}=0, a_{24,16}=0, a_{24,17}=0, a_{24,18}=1, a_{18,29}=0, a_{17,22}=1, a_{17,23}= \\
0, a_{17,24}=0, a_{17,25}=0, a_{18,22}=0, a_{19,22}=0, a_{20,22}=1, a_{21,22}=1\end{array}$ \\
\hline 24 & $\begin{array}{l}a_{25,11}=1, a_{21,29}=a_{20,29}, a_{19,22}=0, a_{19,23}=0, a_{19,24}=0, a_{19,25}= \\
\\
0, a_{18,15}=0, a_{18,16}=0, a_{18,17}=0, a_{18,18}=1, a_{19,18}=0, a_{20,18}=0, a_{22,18}= \\
0\end{array}$ \\
\hline 25 & $\begin{array}{l}a_{19,11}=0, a_{20,15}=0, a_{20,16}=0, a_{20,17}=0, a_{20,18}=0, a_{22,22}= \\
\\
a_{21,22}, a_{22,23}=a_{21,23}, a_{22,24}=a_{21,24}, a_{22,25}=a_{21,25}, a_{23,29}=0\end{array}$ \\
\hline 26 & $\begin{array}{l}a_{21,11}=0, a_{23,15}=a_{22,15}, a_{23,16}=a_{22,16}, a_{23,17}=a_{22,17}, a_{23,18}= \\
a_{22,18}, a_{24,22}=0, a_{24,23}=0, a_{24,24}=0, a_{24,25}=0, a_{24,29}=1,\end{array}$ \\
\hline 27 & $\begin{array}{l}a_{24,11}=a_{23,11}, a_{25,15}=0, a_{25,16}=0, a_{25,17}=0, a_{25,18}=0, a_{25,22}= \\
1, a_{25,23}=1, a_{25,24}=1, a_{25,25}=1, a_{26,29}=0\end{array}$ \\
\hline 28 & $\begin{array}{l}a_{26,11}=0, a_{26,15}=1, a_{26,16}=1, a_{26,17}=1, a_{26,18}=1, a_{27,22}=0, a_{27,23}= \\
0, a_{27,24}=0, a_{27,25}=0, a_{28,29}=0\end{array}$ \\
\hline 29 & $\begin{array}{l}a_{27,11}=1, a_{28,15}=0, a_{28,16}=0, a_{28,17}=0, a_{28,18}=0, a_{29,22}=0, a_{29,23}= \\
\\
0, a_{29,24}=0, a_{24,25}=0, a_{25,25}=1, a_{27,25}=0, a_{29,25}=1\end{array}$ \\
\hline 30 & $\begin{array}{l}a_{29,11}=0, a_{30,15}=0, a_{30,16}=0, a_{30,17}=0, a_{30,18}=1, a_{25,18}=0, a_{26,18}= \\
1, a_{28,18}=0\end{array}$ \\
\hline 31 & $a_{31,11}=1, a_{26,11}=0, a_{27,11}=1, a_{29,11}=0$ \\
\hline 95 & $a_{92,32}=1$ \\
\hline
\end{tabular}

Table 8. Sufficient conditions on $a_{i}$ for 21-95 rounds of the differential path in Table 5. 